\title{
Duplex perception with musical stimuli: A further investigation
}

\author{
SYLVIE C. COLLINS \\ University of Manchester, Manchester, England
}

\begin{abstract}
Duplex perception, the simultaneous perception of a single stimulus in two phenomenologically distinct modes, was first observed, using musical stimuli, by Pastore et al. (1983). The present paper reports a two-part experiment designed to replicate and extend Pastore et al.'s study. Twenty subjects participated in both parts, the first of which was a modified replication of Pastore et al.'s Experiment 1, and the second of which was an extended replication in which the onset time of target notes was manipulated. As expected, in both parts of the study duplex perception was observed only when subjects were making musical judgments of dichotic stimuli; however, in the second part of the study, the prediction that duplex perception would be more likely to occur when target notes were on time as opposed to early or late was not supported. The overall incidence of duplex perception was lower than that reported by Pastore et al. Possible reasons for this discrepancy in findings are discussed.
\end{abstract}

Duplex perception, the simultaneous perception of a single stimulus in two phenomenologically distinct modes, was first observed by Rand (1974), using synthetic speech stimuli. The phenomenon occurs when a single formant transition, or a pair of transitions, is isolated and presented to one ear, while the remainder of the syllable is presented to the other ear. Listeners report hearing a nonspeech "chirp" in the ear to which the isolated transition(s) was (were) presented and, simultaneously, the reconstituted syllable in the other ear. The perceived identity of the syllable-consonant depends on the contralateral formant transition.

Mann, Madden, Russell, and Liberman (1981) applied this method to determine how a time-varying formant transition was integrated into the perception of a stop consonant. They observed that the reconstituted speech percept observed by their subjects seemed no different from the one that would have been produced had the base and isolated transitions been electronically mixed and presented in the normal manner. At the same time, their subjects perceived a nonspeech "chirp" not perceptibly different from what they would have perceived had the transition been presented in isolation. Isenberg and Liberman (1978) used only the F3 transition as the isolated cue, so that the remainder, all of the first and second formants, would be full and speech-like. The results indicated that this arrangement did make it relatively easy to obtain the duplex percept.

There has since been much research using the duplex paradigm (e.g., Isenberg \& Liberman, 1978; Liberman,

The author is grateful to Ian Christensen, Andrew Gregory, Tony Manstead, and two anonymous reviewers for their helpful comments on an earlier version of this paper.

The author's mailing address is: Department of Psychology, University of Manchester, Manchester M13 9PL, England.
Isenberg, \& Rakerd, 1981; Mann, 1980; Mann et al., 1981 ; Nusbaum, Schwab, \& Sawusch, 1983; Repp, Milburn, \& Ashkenas, 1983). All of these studies focused on duplex perception in the context of investigating certain properties of human speech perception. Few researchers have attempted any thorough explanation of the phenomenon (however, see Nusbaum, 1984; Nusbaum et al., 1983; Repp, 1984; Repp et al., 1983), and until very recently no one has attempted to apply the paradigm to another area of auditory perception. If the phenomenon were observed in other areas of auditory perception, this would imply that duplex perception is part of a generalized auditory process rather than being special to speech.

Pastore, Schmuckler, Rosenblum, and Szczesiul (1983) used musical stimuli in two experiments intended to demonstrate duplex perception. In the first experiment, major and minor triads were produced by dichotic fusion of two simultaneous piano notes presented to one ear (perfect 5th) with a "natural" or "flat" single note presented to the opposite ear. It was found that musically trained subjects perceived simultaneously both the single tone and a fused (major or minor) chord. The labeling of chords as major or minor was more accurate than the labeling of single notes as natural or flat.

In the second experiment, which used pure tones in place of piano tones, another sample of musically trained subjects individually exhibited categorical perception for either the fused chord or the single tone but never for both types of stimuli.

According to Pastore et al. (1983), "duplex perception is based upon the notion that a given stimulus yields different results when processed in different perceptual modes" (p. 472). In their study, Pastore et al. required subjects to make judgments in two modes, musical and acoustical. They used three types of stimulus presentation: monaural presentation of chords, diotic presentation 
of single notes, and dichotic presentation of an interval of a perfect 5th to one ear and the middle note of the chord to the other. The subjects were required to identify the monaural chords as major or minor, the diotic stimuli as natural or flat, and to make both kinds of judgments for the dichotic stimuli, that is, in one condition to identify the chord as major or minor and in another condition to identify the middle note of the chord as natural or flat. The task of identifying the single note as natural or flat was classified as an acoustical task. The problem with such a classification is that when subjects are asked to label the middle note of a chord as natural or flat, they may first make a musical judgment (i.e., whether the chord is major or minor) and then decide whether the target note is natural or flat. Recognizing this problem, Pastore et al. attempted to force subjects to make purely acoustical judgments of dichotic as well as diotic stimuli by requiring them to make natural/flat judgments of both types of stimuli within the same condition. Rather surprisingly, this strategy appears to have worked, in that Pastore et al. found that natural/flat judgments of the middle note of dichotically presented triads were no more accurate (indeed, were, on average, less accurate) than natural/flat judgments of single notes presented diotically.

One might question whether the effect of mixing diotic and dichotic stimulus presentations would simply have been to focus subjects' attention on the acoustical processing of stimuli. One could equally well reason that the musical context supplied by the intermittent presentation of the perfect 5th to the nontarget ear (in the dichotic conditions) would actually promote musical processing, since the presence of the interval would be highlighted by virtue of the fact that it did not appear on each presentation. Furthermore, the dichotically presented triads would have provided reference notes with which adjacent diotically presented single notes could be compared. For a note to be judged natural or flat, it must be compared with a standard; the standard may be external, in the shape of an adjacent chord, or internal, in the shape of a wellcalibrated memory for pitch. Judging the naturalness/flatness of diotically presented single notes that are interspersed with dichotically presented chords should, therefore, be rather easier than making similar judgments of a series of diotically presented single notes. However, the same reasoning suggests that judging whether a note is natural or flat should be easier when accompanied by an interval than when judged in isolation, yet Pastore et al. found no difference in judgmental accuracy. It may be that the relatively poor judgments of dichotically presented stimuli observed by Pastore et al. arose from a confusion in subjects, engendered by the inconsistent nature of the stimuli they were required to judge. In other words, the apparent failure of subjects to make use of the contextual information provided by the interval may simply have reflected a disruption of musical processing, rather than a promotion of acoustical processing. However, it should be noted that this point applies only to Pastore et al.'s Experiment 1 and not to their Experiment 2, since, in the latter, triads and single notes were judged in separate blocks of trials. In some respects, then, the conditions used in the present study resemble quite closely those in Pastore et al.'s Experiment 2.

In the first part of the present study, diotic and dichotic stimuli were presented in separate conditions. Subjects were required to judge the notes presented in the diotic condition as natural or flat-acoustical judgments. There were two conditions in which stimuli were presented dichotically, with the interval of a perfect 5 th presented to one ear and the middle note of the triad to the other; in one of these conditions, subjects were required to make an acoustical judgment of the middle note of the triad. In addition, these same acoustical judgments were required in relation to the middle note of some monaurally presented triads. Thus, natural/flat judgments should be approximately equal in accuracy across these three types of presentation if all stimuli were processed acoustically, but dichotic and monaural presentations should result in superior accuracy if the stimuli were processed musically as a chord, with natural/flat judgments inferred from the musical judgment. It is interesting to note that the order of judgments conjectured here is the reverse of that conjectured for speech stimuli by Nusbaum et al. (1983).

Subjects were also asked to make musical judgments in relation to other monaurally and dichotically presented triads, that is, to judge whether the chord was major or minor. Following Pastore et al., it was predicted that subjects would be more accurate in labeling chords as major or minor than in labeling single notes as natural or flat. Finally, subjects were asked to judge, in relation to each stimulus presentation, how many notes had been presented to each ear. These judgments were used to identify instances of duplex perception, that is, simultaneously hearing the complete chord and the single tone. Following Pastore et al., it was predicted that, when making musical judgments of dichotically presented stimuli, subjects would consistently hear both the complete chord and the single tone.

In the second part of the present study, the onset time of the target note was varied such that it was presented $125 \mathrm{msec}$ before, simultaneously with, or $125 \mathrm{msec}$ after the rest of the stimulus. In other respects, the stimuli and the manner in which they were presented (diotically, dichotically, or monaurally) were the same as in the first part. Here, subjects were required to make "acoustical" judgments in relation to each stimulus presentation, by assessing whether the target tone was early, on time, or late. These judgments were expected to be equally accurate across the three types of presentation, since each contained a referent, in the form of a tone or pair of tones, with respect to which the onset time of the target tone could be judged. Clearly, such timing judgments are easier to make than are the natural/flat judgments required in Part 1 of the study. Assuming that subjects would be reasonably accurate in their timing judgments, it was anticipated that varying onset time would have an impact on the incidence of duplex perception. This is because when 
the target note is late, the interval alone is presented for $125 \mathrm{msec}$, and one would therefore expect subjects to hear clearly that there was only an interval of a perfect 5 th rather than a whole triad. Wallach, Newman, and Rosenzweig's (1949) study shows that the upper limit of the time interval over which fusion of separate sound stimuli takes place is about $40 \mathrm{msec}$ for complex stimuli such as speech or music. Thus, $125 \mathrm{msec}$ should be a sufficient interval for the signals to the different ears to remain independent of each other. When the target note is early, one would expect a certain amount of confusion as to whether the second part of the stimulus was an interval or a triad, since the single note is still present during the presentation of the interval. When the target note is on time, one would expect the responses to mimic those of the first part of the study. Thus, the tendency to hear both the complete chord and the single note when listening to dichotically presented stimuli should be reduced when the target note is early or late.

The subjects were all students of music with substantial aural training experience. After hearing an example of each type of stimulus presentation, each subject was given a practice session in order to become accustomed to the task requirements. Three musical keys were used further to reduce the possibility that natural/flat judgments were mediated by intraexperiment memory for pitch. Computer-generated tones were used throughout.

\section{METHOD}

\section{Subjects}

Twenty undergraduate students from the Faculty of Music, University of Manchester, participated. Each subject had achieved diploma qualifications in at least one instrument and had received regular advanced aural training for a period of at least 3 years.

\section{Stimuli}

All stimuli were pure tones produced by Wavetek 132 VCG and Farnell F 0765.007 generators (12-bit precision, $8000-\mathrm{Hz}$ sampling rate), and were band-pass filtered (250-700 Hz) through a Kemo $0.01 \mathrm{~Hz}-100 \mathrm{kHz}$ dual variable filter, Type VBF/8. The stimuli were presented as single notes or in groups of three notes, in major and minor triads. The frequencies used, derived from the natural scale, were:

$$
\begin{array}{ll}
264,315.5 / 330,396 \mathrm{~Hz} & \text { (c, eb/eq, g) } \\
330,396 / 418,495 \mathrm{~Hz} & (\mathrm{e}, \mathrm{g} q / \mathrm{g} \sharp, \text { b) } \\
390,467.5 / 495,594 \mathrm{~Hz} & (\mathrm{~g}, \mathrm{bb} / \mathrm{b} \sharp, \text { d })
\end{array}
$$

All stimuli were $500 \mathrm{msec}$ in duration, including $12-\mathrm{msec}$ onset and offset times. Tape 1, used in the first part of the study, consisted of the following stimuli: (1) 78 monaural presentations of triads (18 practice and 60 experimental), (2) 120 dichotic presentations of triads-one note to one ear and two to the other (6 practice and 90 experimental), and (3) 54 diotic presentations of the same note to both ears (6 practice and 48 experimental).

Tape 2, used in the second part of the study, consisted of the following stimuli: (1) 72 monaural presentations of triads (12 practice and 60 experimental), (2) 96 dichotic presentations of triads (6 practice and 90 experimental), and (3) 66 diotic presentations of single notes ( 6 practice and 60 experimental). The onset of the stimulus tone to the target ear was varied with respect to the onset time of the rest of the stimulus to the other ear, except in the monaural condition, in which the onset of the middle note of the triad was varied with respect to the rest of the stimulus to that same ear. The target tones were presented $125 \mathrm{msec}$ before (early), $125 \mathrm{msec}$ after (late), or simultaneously with (on time) the rest of the stimulus. In each presentation, the signals ended simultaneously.

There were equal numbers of the different frequency combinations and onset patterns on the respective tapes. The stimuli were presented in random sequences, with interstimulus intervals of $5 \mathrm{sec}$ and intercondition intervals of $7 \mathrm{sec}$. The signals were presented at $70 \mathrm{~dB} \mathrm{SPL}$.

\section{Procedure}

The hearing of each subject was tested using a Grason-Stadler 1707 audiometer. None showed a hearing loss of more than $3 \mathrm{~dB}$ for frequencies between 250 and $700 \mathrm{~Hz}$ (the range of frequencies used in the experiment). The experiment was run in a soundproof chamber, and the subjects were tested individually. For all the stimuli, the subjects were required to record how many notes they had heard in each ear. For Tape 1, the subjects were required to make acoustical (natural/flat) judgments of all the stimuli in the diotic condition (diotic/acoustical condition) and all of the stimuli in one of the two dichotic conditions (dichotic/acoustical condition) and one of the two monaural conditions (monaural/acoustical condition). The subjects were required to make musical (major/minor) judgments of the stimuli in the second dichotic (dichotic/musical condition) and second monaural (monaural/musical condition) conditions. For Tape 2, the subjects were required to judge whether the triad was major or minor in all the monaural and dichotic stimuli. In addition, the subjects were asked to judge whether the target note of each stimulus was early, on time, or late, relative to the rest of the stimulus. The subjects listened to examples of all the stimuli and then completed a practice run in order to familiarize themselves with the stimuli and the response sheets. The two tapes were run in the same session with an interval of $5 \mathrm{~min}$ between tapes; the whole session lasted approximately $1 \mathrm{~h}$. Target ear and order of presentation of tapes were counterbalanced. The target ear was that to which the isolated note or middle note of the triadic stimuli was presented.

\section{Scoring}

All responses were scored as correct or incorrect. The three different types of response were scored separately. Particular attention was paid to those responses in which subjects reported hearing more notes than were physically present; responses in which subjects reported hearing one note to one ear and three to the other were noted as instances of duplex perception.

\section{Analysis}

Analysis was carried out using a series of one-way and two-way analyses of variance, with type of judgment (musical/acoustical), type of presentation (monaural/diotic/dichotic), and (where appropriate) onset time (early/on time/late) as the factors. The data entered into these analyses were percentage correct scores to which an arcsine transformation had been applied.

\section{RESULTS}

\section{Tape 1}

The mean percent correct scores for all conditions are presented in Table 1. When accuracy scores were entered into a two-way analysis of variance using presentation (monaural vs. dichotic) and judgment type (musical vs. acoustical) as factors, main effects were found for both presentation $[\mathrm{F}(1,19)=21.56, \mathrm{p}<.01]$ and judgment 
Table 1

Tape 1: Mean Percent Correct Scores for All Subjects

\begin{tabular}{lcccc}
\hline & \multicolumn{3}{c}{ Presentation } & \\
\cline { 2 - 4 } Judgment Type & Monaural & Dichotic & Diotic & Row Means \\
\hline Acoustical & 67.90 & 82.85 & 61.95 & 70.90 \\
Musical & 80.90 & 88.50 & & 84.70 \\
Column Means & 74.40 & 85.68 & 61.95 & 77.80 \\
\hline
\end{tabular}

Note-These figures were obtained by calculating the number of correct responses as a percentage of the total number of stimuli presented for each condition to each subject.

Table 2

Tape 1: Mean Percent Occurrence of Reports of Hearing More Than Three Notes

\begin{tabular}{|c|c|c|}
\hline Sample & $\begin{array}{l}\text { Overestimates as } \\
\text { Proportion of } \\
\text { All Judgments }\end{array}$ & $\begin{array}{c}\text { Proportion } \\
\text { Overestimates } \\
\text { in Duplex Format }\end{array}$ \\
\hline All subjects & 27.16 & 40.90 \\
\hline Overestimators Only & 43.00 & 61.92 \\
\hline
\end{tabular}

Note - The first row reports the number of judgments that were overestimates as a percentage of all judgments made in the dichotic/musical condition. The second row reports the equivalent figures as a percentage of the judgments made in this condition by subjects who overestimated the number of notes on at least one occasion.

type $[\mathrm{F}(1,19)=16.79, \mathrm{p}<.01]$. Accuracy was greater in the dichotic presentation than in the monaural presentation. As expected, musical judgments were more accurate than acoustical judgments. Scheffé tests indicated that monaural/musical judgments (mean $=80.90 \%$ ) were significantly $(\mathrm{p}<.01)$ more accurate than monaural/ acoustical judgments (mean $=67.90 \%$ ), but that dichotic/musical judgments (mean $=88.50 \%$ ) were not significantly more accurate than dichotic/acoustical judgments (mean $=82.85 \%$ ). Analysis of the acoustical judgments revealed a significant effect due to presentation $[F(2,38)=14.88, p<.01]$. Scheffé tests showed that natural/flat judgments were significantly more accurate in the dichotic condition (mean $=82.85 \%$ ) than in the diotic condition (mean $=61.95 \%$ ) or in the monaural condition (mean $=67.90 \%)(p<.01$ in each case).

As predicted, the only condition in which subjects reported hearing more than three notes was the dichotic/ musical condition. The mean percent occurrences of such judgments are displayed in Table 2 . Seven of the 20 subjects never reported hearing more than three notes, that is, there was no sign of their having heard the duplex phenomenon. Only three subjects reported duplex percepts throughout the dichotic/musical condition, that is, throughout that condition these subjects heard the isolated single note and the fused chord in the contralateral ear.

\section{Tape 2}

The mean percent correct scores for all conditions are presented in Table 3. As expected, the acoustical judgments revealed no significant effects due to onset time or presentation [onset main effect, $\mathrm{F}(2,38)=3.43$, n.s.; presentation main effect, $F(1,19)=.87$, n.s.].
Similar analysis of the musical judgments revealed a significant effect due to onset time $[F(2,38)=13.76$, $\mathrm{p}<.01]$. Scheffé tests showed that judgments were significantly more accurate when the target note was on time $($ mean $=89.05 \%)$ than when it was early (mean $=$ $71.80 \%)$ or late $($ mean $=74.13 \%)(\mathrm{p}<.01$ and $\mathrm{p}<$ .05 , respectively). Neither the main effect of presentation nor the interaction between presentation and onset time had significant effects on musical judgments.

Again, the only condition in which subjects reported hearing more than three notes was the dichotic/musical condition. The mean percent occurrences of such judgments are shown in Table 4 . Eight of the 20 subjects never reported hearing more than three notes. Only 5 of the 20 subjects consistently reported hearing four notes in duplex format. Subjects did more often report hearing more than three notes when the target note was on time (mean = $28.66 \%$ ) than when it was early (mean $=18.95 \%$ ) or late (mean $=12.18 \%$ ); however, this difference was not significant. There was no significant effect of onset time on the number of duplex percepts reported.

Ear of presentation had no significant impact on any of the measures in relation to either tape. For both tapes, when subjects reported hearing more than three notes but did not report a duplex percept, they reported hearing two notes to both ears. This may simply have been misassignment of stimuli to interval and target note ears due to the number of analytic judgments the subjects were required to make.

\section{DISCUSSION}

In the first part of the study, the prediction that subjects would have greater difficulty in labeling isolated single notes than in labeling the musical relationships was supported. Subjects' musical judgments were significantly more accurate than their acoustical judgments. This result is consistent with Pastore et al.'s findings. As those authors point out, it is not surprising that judgments that involve a stable external reference (in this case the perfect 5 th) are more accurate than judgments of stimuli in isolation. The superior accuracy of musical judgments

Table 3

Tape 2: Mean Percent Correct Scores for All Subjects

\begin{tabular}{|c|c|c|c|c|c|}
\hline \multirow{2}{*}{$\begin{array}{l}\text { Judgment } \\
\text { Type }\end{array}$} & \multirow{2}{*}{$\begin{array}{l}\text { Onset } \\
\text { Time }\end{array}$} & \multicolumn{3}{|c|}{ Presentation } & \multirow[b]{2}{*}{ Row Means } \\
\hline & & Monaural & Dichotic & Diotic & \\
\hline Acoustical & $\begin{array}{l}\text { Early } \\
\text { On Time } \\
\text { Late } \\
\text { Mean }\end{array}$ & $\begin{array}{l}81.25 \\
81.25 \\
67.50 \\
76.67\end{array}$ & $\begin{array}{l}72.23 \\
88.97 \\
79.05 \\
80.08\end{array}$ & $\begin{array}{l}68.90 \\
84.90 \\
74.10 \\
75.97\end{array}$ & $\begin{array}{l}74.13 \\
85.04 \\
73.55 \\
77.57\end{array}$ \\
\hline Muscial & $\begin{array}{l}\text { Early } \\
\text { On Time } \\
\text { Late } \\
\text { Mean }\end{array}$ & $\begin{array}{l}70.65 \\
88.10 \\
72.00 \\
76.92\end{array}$ & $\begin{array}{l}72.95 \\
90.00 \\
76.25 \\
79.73\end{array}$ & & $\begin{array}{l}71.80 \\
89.05 \\
74.13 \\
78.33\end{array}$ \\
\hline
\end{tabular}

Note-These figures were obtained by calculating the number of correct responses as a percentage of the total number of stimuli presented for each condition to each subject. 
Table 4

Tape 2: Mean Percent Occurrence of Subjects Reporting Hearing More Than Three Notes

\begin{tabular}{llcccc}
\hline & \multicolumn{1}{c}{$\begin{array}{c}\text { Measure } \\
\text { Sample }\end{array}$} & Early & On Time & Late & Row Means \\
\hline All Subjects & $\begin{array}{l}\text { Overestimates as Percent } \\
\text { of All Judgments }\end{array}$ & 18.95 & 28.66 & 12.18 & 19.19 \\
& $\begin{array}{l}\text { Percent of Overestimates } \\
\text { in Duplex Format }\end{array}$ & 33.55 & 33.18 & 34.61 & 33.78 \\
Overestimators Only & $\begin{array}{l}\text { Overestimates as Percent } \\
\text { of All Judgments }\end{array}$ & 37.90 & 52.95 & 25.40 & 38.75 \\
& $\begin{array}{l}\text { Percent of Overestimates } \\
\text { in Duplex Format }\end{array}$ & 70.28 & 78.81 & 62.62 & 70.57 \\
\hline
\end{tabular}

Note-The first row reports the number of judgments that were overestimates as a percentage of all judgments made in the dichotic/muscial condition. The second row reports the equivalent figures as a percentage of the judgments made in this condition by subjects who overestimated the number of notes heard on at least one presentation.

over acoustical judgments varied across presentations. The monaural/musical and dichotic/musical judgments were both considerably more accurate than the monaural/ acoustical or diotic/acoustical judgments. However, the dichotic/acoustical judgments were, on average, quite accurate, and were not significantly less accurate than the dichotic/musical judgments. This is a puzzling pattern of findings, because one cannot account for the accuracy of the dichotic/acoustical judgments simply in terms of the impact of the presence of the two other notes of the chord, since these two notes were also present in the monaural/ acoustical stimuli. Moreover, it seems unlikely that the dichotic/acoustical judgments were simply the result of "musical" processing, because the fact that duplex percepts were confined to the dichotic/musical condition suggests that the subjects did use different perceptual modes when making musical and acoustical judgments of dichotically presented stimuli.

There was some support for the prediction that subjects would simultaneously hear the single note and the complete chord. As already noted, this occurred only in the musical/dichotic condition; $27.16 \%$ of responses in this condition indicated that subjects had overestimated the number of notes heard. However, only $40 \%$ of these response were in duplex format. Thus, only $11.10 \%$ of all the responses in this condition were in duplex format, although Pastore et al. reported $100 \%$.

In the second part of the study, the only condition in which duplex perception occurred was again the musical/dichotic condition: here $19 \%$ of responses indicated that the subjects had overestimated the number of notes present. However, only $33.78 \%$ of these were in duplex format; thus, only $6.48 \%$ of all the responses in this condition were in duplex format. The difference in the incidence of duplex responses between this experiment and the first is 4.62 percentage points $[t(38)=1.93$, n.s.]. When the target notes of the musical/dichotic stimuli were early or late, this had the expected effect of attenuating the tendency for subjects to judge that more than three notes had been presented, but this tendency fell short of statistical significance.

Clearly, the most noticeable difference between this study and that of Pastore et al. is the extent to which duplex percepts are reported by subjects. The results of the two studies differ by a factor of 10 in this respect. This suggests that Pastore et al.'s results exaggerate the incidence of duplex perception in the musical population. It is worth noting in this connection that Pastore et al. did discard $25 \%$ of their subjects for (1) failing to exceed chance performance and (2) failing to label the stimuli. It may be that in discarding these subjects the investigators inadvertently eliminated subjects who would not have exhibited duplex perception.

However, the present study used a very direct measure of the incidence of duplex perception, namely, asking subjects how many notes they had heard. Direct comparison with the Pastore et al. findings is difficult in view of the fact that the latter investigators did not employ direct measures. It might be argued that one can infer the incidence of duplex perception in Pastore et al.'s study by assuming that subjects who correctly identified the dichotic chords with $90 \%-100 \%$ accuracy were "always" perceiving duplex percepts, whereas those with less than $90 \%$ accuracy for the chords but superior accuracy for chords over notes were "sometimes" perceiving duplex percepts. Applying these criteria to the results of Pastore et al.'s Experiment 1,35\% of the subjects meet the criterion for always perceiving duplex percepts and $40 \%$ did so for sometimes; the remaining $25 \%$ could not reliably perceive duplex percepts. This admittedly rather arbitrary analysis makes the incidence of duplex perception in Pastore et al.'s results more consistent with the figures obtained by the more direct measure used in the present study.

The overall incidence of duplex perception in the present study is very low, but if it were simply due to chance, one would expect a more random distribution across subjects. As it was, 13 of the 20 subjects reported hearing four notes in the musical/dichotic condition and 7 of these 
subjects consistently reported these percepts as duplex in form. Furthermore, 2 subjects consistently reported duplex percepts throughout the musical/dichotic conditions in both parts of the study. They both claimed to have "perfect pitch," and, indeed, showed almost perfect accuracy in both musical and acoustical judgments throughout the testing. However, there was another subject who gave a perfect performance on musical and acoustical judgments throughout, without claiming to have perfect pitch, and who never once reported hearing a duplex percept! The wide range of responses is possibly due to individual differences in perceptual analysis. One would expect subjects who analyze the stimuli holistically to exhibit duplex perception more often than those who analyze analytically. It may be that the low overall incidence of duplex perception is due in part to the types of analyses required of the subjects. Subjects were first asked to identify the number of notes perceived and then asked to make a musical judgment, that is, subjects were first required to respond analytically and then holistically. Requiring subjects to make analytic judgments first may have mitigated any tendency on their part to make holistic analyses, thereby decreasing the likelihood of duplex percepts being observed.

Finally, despite the low incidence of duplex perception in the present study, it is clear that the phenomenon exists with musical stimuli and therefore cannot be taken as evidence for the operation of a specialized phonetic mode. The present results do, to an extent, support Pastore et al.'s insight that duplex perception may represent a more general context effect in auditory perception. However, there is patently a need to conduct further research addressing the question of individual differences in the tendency to hear dichotically presented musical triads in duplex format.

\section{REFERENCES}

Isenberg, D. S., \& Liberman, A. M. (1978). Speech and non-speech percepts from the same sound. Journal of the Acoustical Society of America, 64 (Suppl. 1), S20. (Abstract)

Liberman, A. M., Isenberg, D., \& Rakerd, B. (1981). Duplex perception of cues for stop consonants: Evidence for a phonetic mode. Perception \& Psychophysics, 30, 133-143.

MANN, V. A. (1980). Influence of preceding liquid on stop-consonant perception. Perception \& Psychophysics, 28, 407-412.

Mann, V. A., Madden, J., Russell, J. M., \& Liberman, A. M. (1981). Further investigations into the influence of preceding liquids on stop consonant perception. Journal of the Acoustical Society of America, 69, S91. (Abstract)

Nusbaum, H. C. (1984). Possible mechanisms of duplex perception: " 'Chirp." identification versus dichotic fusion. Perception \& Psychophysics, 35, 94-101.

Nusbaum, H. C., Schwab, E. C., \& Sawusch, J. R. (1983). The role of "chirp" identification in duplex perception. Perception \& Psychophysics, 33, 323-332.

Pastore, R. E., Schmuckler, M. A., Rosenblum, L., \& Szczesiul, R. (1983). Duplex perception with musical stimuli. Perception \& Psychophysics, 33, 469-474.

RAND, T. C. (1974). Dichotic release from masking for speech. Journal of the Acoustical Society of America, 55, 678-680.

REPP, B. H. (1984). Against a role of "chirp" identification in duplex perception. Perception \& Psychophysics, 35, 89-93.

Repp, B. H., Milburn, C., \& Ashkenas, J. (1983). Duplex perception: Confirmation of fusion. Perception \& Psychophysics, 33, 333-337.

Wallach, H., Newman, E. B., \& Rosenzweig, M. R. (1949). The precedence effect in sound localization. American Journal of Psychology, 62, 315-336.

\section{NOTE}

1. The author would like to thank an anonymous reviewer for this helpful suggestion.

(Manuscript received November 2, 1984; revision accepted for publication July $16,1985$. 